\title{
AVERAGE GOLDBACH AND THE QUASI-RIEMANN HYPOTHESIS
}

\author{
GAUTAMI BHOWMIK AND IMRE Z. RUZSA
}

\begin{abstract}
We prove that a good average order on the Goldbach generating function implies that the real parts of the non-trivial zeros of the Riemann zeta function are strictly less than 1. This together with existing results establishes an equivalence between such asymptotics and the Riemann Hypothesis.
\end{abstract}

\section{INTRODUCTION}

The relation between the average order of the Goldbach generating function and the Riemann Hypothesis was introduced by Granville [4. In an attempt to generalise such relations to sums of primes in arithmetic progressions it was observed that asymptotic results on the average number of restricted representations of integers assuming a conjecture on distinct zeros of Dirichlet L-functions did not obviously yield an equivalence with the generalised Riemann Hypothesis since the case of the real part of non-trivial zeros being exactly 1 could not be eliminated. In this note we succeed in doing so for the Riemann zeta function in a simple way and establish an equivalence of average orders of the original Goldbach function with what we call a quasi-Riemann hypothesis, that the real parts of the non-trivial zeros of of the Riemann zeta function are strictly less than 1. This completes the proof of Theorem 1A in [4] i.e. a good asymptotic order of the Goldbach function is indeed equivalent to the Riemann Hypothesis. The same idea has since been applied to the restricted sums of two primes both in the same congruence class (Section 8 [1]).

The average order of the function

$$
G(n)=\sum_{k_{1}+k_{2}=n} \Lambda\left(k_{1}\right) \Lambda\left(k_{2}\right)
$$

where $\Lambda$ is the von Mangoldt function was studied by Fujii [3] and Granville (op.cit) who showed that under the Riemann Hypothesis the summatory function can be expressed as

$$
S(x)=\sum_{n \leq x} G(n)=\frac{x^{2}}{2}+O\left(x^{3 / 2}\right) .
$$

The error term was eventually improved to its conjectured value $O\left(x^{1+\varepsilon}\right)$ [2] but here it is enough for us to assume any error term less than $x^{2}$ and from the square root of the associated power series estimate well the Chebyshev function, $\psi(x)=\sum_{n \leq x} \Lambda(n)$, using a Dirichlet kernel.

2010 Mathematics Subject Classification. 11P32, 11M26.

Key words and phrases. Goldbach problem, Riemann hypothesis, Chebyshev Function, Dirichlet Kernel.

The second author was supported by ERC-AdG Grant No.321104 and Hungarian National Foundation for Scientific Research (OTKA), Grants No.109789 and NK104183. 


\section{Quasi-Riemann Hypothesis}

Theorem 2.1. If the asymptotic relation

$$
S(x)=\frac{x^{2}}{2}+O\left(x^{2-\delta}\right), \delta>0
$$

holds, then there exists $0<\delta^{\prime}<1$ such that for the non-trivial zeros $\rho$ of the Riemann zeta function $\operatorname{Re} \rho<1-\delta^{\prime}$.

Proof. We consider the power series

$$
F(z)=\sum_{n=1}^{\infty} \Lambda(n) z^{n},|z|<1
$$

whose square is written in terms of the Goldbach function as

$$
F(z)^{2}=\sum_{n=1}^{\infty} G(n) z^{n} .
$$

Writing the above as a telescoping series with $G(n)=S(n)-S(n-1)$ we get

$$
F(z)^{2}=(1-z) \sum_{n=1}^{\infty} S(n) z^{n} .
$$

From the assumption (2.1) on the Goldbach asymptotic order we infer

$$
\sum_{n=1}^{\infty} S(n) z^{n}=\sum_{n}\left(\frac{n^{2}}{2}+O\left(n^{2-\delta}\right)\right) z^{n}=\sum_{n} \frac{n^{2}}{2} z^{n}+O\left(\sum_{n} n^{2-\delta}|z|^{n}\right) .
$$

We have

$$
\sum_{n} \frac{n^{2}}{2} z^{n}=\frac{1}{(1-z)^{3}}-\frac{3}{2(1-z)^{2}}+\frac{1}{2(1-z)}
$$

while the error term of (2.2) is estimated as

$$
\sum_{n=1}^{\infty} n^{2-\delta}|z|^{n} \ll(1-|z|)^{\delta-3}
$$

by comparison with the power series expansion of the right hand side and thus

$$
\sum_{n} S(n) z^{n}=\frac{1}{(1-z)^{3}}+O\left(\frac{1}{(1-|z|)^{3-\delta}}\right)
$$

which implies

$$
F(z)^{2}=(1-z) \sum_{n=1}^{\infty} S(n) z^{n}=\frac{1}{(1-z)^{2}}+O\left(\frac{|1-z|}{(1-|z|)^{3-\delta}}\right) .
$$

To estimate $\psi(N)$ we consider the circle $|z|=R=1-1 / N$ for a large positive integer $N$ and rewrite the last formula as

$$
F(z)^{2}=\frac{1}{(1-z)^{2}}+O\left(|1-z| N^{3-\delta}\right) .
$$

The error term is less than the absolute value of the main term for

$$
|1-z|<N^{\frac{\delta}{3}-1}
$$


and in this range we take the complex square root of (2.3) to obtain

$$
F(z)=\frac{1}{1-z}+O\left(|1-z|^{2} N^{3-\delta}\right) .
$$

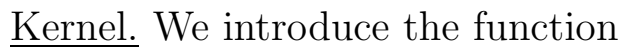

$$
K(z)=z^{-N-1} \frac{1-z^{N}}{1-z} .
$$

We have $K(z) \ll|1-z|^{-1}$ and we use it to estimate the partial sums

$$
\psi(N)=\frac{1}{2 \pi i} \int_{|z|=R} F(z) K(z) d z=N+\frac{1}{2 \pi i} \int_{|z|=R}\left(F(z)-\frac{1}{1-z}\right) K(z) d z .
$$

Major Arc. On the part of the circle (2.4) the integrand is

$$
O\left(|1-z| N^{3-\delta}\right)=O\left(N^{2-2 \delta / 3}\right)
$$

so the contribution to the integral above is $O\left(N^{1-\delta / 3}\right)$ from the power series estimate obtained in (2.5).

Minor Arc. On the rest of the circle, i.e. the arc $|1-z|>N^{\delta / 3-1}$ we use the CauchySchwarz inequality. For $F$ we apply the estimate for the full circle

$$
\begin{aligned}
\int_{|z|=R}\left|F(z)-\frac{1}{1-z}\right|^{2}|d z| & =\int_{0}^{2 \pi} \sum_{n}(\Lambda(n)-1) R^{n} e^{i n t} \sum_{m}(\Lambda(n)-1) R^{n} e^{-i m t} \\
& =2 \pi \sum_{n=1}^{\infty}(\Lambda(n)-1)^{2}\left(1-\frac{1}{N}\right)^{2 n}=O(N \log N) .
\end{aligned}
$$

We estimate the square integral of the kernel as follows.

$$
I=\int_{\substack{|z|=R \\|1-z|>N^{\delta / 3-1}}}|K(z)|^{2}|d z| \ll \int_{\substack{|z|=R \\|1-z|>N^{\delta / 3-1}}} \frac{|d z|}{|1-z|^{2}} .
$$

With the parametrization $z=R e^{i t}$ the last integral becomes

$$
2 \int_{t_{0}}^{\pi} \frac{1}{\left|1-R e^{i t}\right|^{2}} d t
$$

where $t_{0}$ is defined by $\left|1-R e^{i t_{0}}\right|=N^{\delta / 3-1}$.

We have

$$
\left|1-R e^{i t}\right|^{2}=1+R^{2}-2 R \cos t=(1-R)^{2}+4 R^{2} \sin ^{2} \frac{t}{2}\left\{\begin{array}{l}
<t^{2}+N^{-2} \\
>t^{2} / 3
\end{array}\right.
$$

whence $t_{0} \gg N^{\delta / 3-1}$ and the integral satisfies

$$
I \ll \int_{t_{0}}^{\pi} t^{-2} d t<t_{0}^{-1} \ll N^{1-\delta / 3} .
$$

Now the Cauchy Schwarz inequality gives the bound for the minor arc as

$$
\left(\int_{|z|=R}\left|F(z)-\frac{1}{(1-z)}\right|^{2} d z\right)^{1 / 2} I^{1 / 2} \ll N^{1-\delta / 6}(\log N)^{1 / 2} .
$$


The major and minor arcs together give

$$
\psi(N)-N \ll N^{1-\delta / 6}(\log N)^{1 / 2},
$$

which proves the theorem with $\delta^{\prime}=\delta / 6$.

\section{REMARKS}

1. Using a better kernel and a more careful calculation we can improve the bound to $\delta^{\prime}=\delta / 3$. It is of little importance in this context as the methods of $[1,2,4]$ give $\delta^{\prime}=\delta$ as soon as we have $\delta^{\prime}<1$, through an explicit expression of $S(x)$ using roots of the zeta-function. We are unable to get $\delta^{\prime}=\delta$ directly by the simple method of this paper and we think it cannot be done. We used very little of the properties of the Liouville function and our method works equally for any function $f$ satisfying $|f(n)|=O\left(n^{\varepsilon}\right)$ for all $\varepsilon>0$ and

$$
\sum_{m+n \leq x} f(m) f(n)=c x^{2}+O\left(x^{2-\delta}\right) .
$$

We doubt that

follows in this generality.

$$
\sum_{n \leq x} f(n)=c^{\prime} x+O\left(x^{1-\delta+o(1)}\right)
$$

2. In general we are yet unable to establish an equivalence between average orders of restricted Goldbach functions and appropriate quasi Riemann Hypotheses for $L$ functions of [4] or [1] though a particular case is handled in [1] using the method of this note.

\section{REFERENCES}

[1] G.Bhowmik, K. Halupczok, K. Matsumoto and Y.Suzuki, Goldbach Representations in Arithmetic Progressions and zeros of Dirichlet $L$-functions, To appear in Mathematika, 2018 , arXiv:1704.06103v1.

[2] G. Bhowmik and J.-C. Schlage-Puchta, Mean representation number of integers as the sum of primes, Nagoya Math. J. 200 (2010), 27-33.

[3] A. Fujii, An additive problem of prime numbers, Acta Arith. 58 (1991), 173-179.

[4] A. Granville, Refinements of Goldbach's conjecture, and the generalized Riemann hypothesis, Funct. Approx. Comment. Math. 37 (2007), 159-173; Corrigendum, ibid. 38 (2008), 235-237.

[5] H. L. Montgomery and R. C. Vaughan, Multiplicative Number Theory I, Classical Theory, Cambridge, 2007.

Laboratoire Paul Painlevé, LabeX-Cempi, Université Lille 1, 59655 Villeneuve d’AscQ Cedex, France

E-mail address: bhowmik@math.univ-lille1.fr

Alfréd Rényi Institute of Mathematics, Budapest, Pf. 127, H-1364 Hungary

E-mail address: ruzsa@renyi.hu 